\title{
A comparison of forward blocking and reduced overshadowing in human causal learning
}

\author{
STEFAAN VANDORPE and JAN DE HOUWER \\ Ghent University, Ghent, Belgium
}

\begin{abstract}
In this study, we directly compared forward blocking with reduced overshadowing in a human causal learning study using an $\mathrm{A}+, \mathrm{B}-$ (first learning stage), $\mathrm{AX}+, \mathrm{BY}+, \mathrm{KL}+$ (second learning stage) design. The results showed that reduced overshadowing was significantly stronger than forward blocking. These results are problematic for at least some associative learning models but were predicted on the basis of higher order reasoning accounts of cue competition in human causal learning.
\end{abstract}

Learning causal relationships is an essential skill in daily life because it allows us to anticipate and control events in the environment. For example, if a person learns that each time he walks in the sun he will get a headache, he will avoid walking in the sun or he will protect his head with a cap. Yet despite the importance of learning causal relationships, there is still much controversy about the underlying processes.

In human causal learning (HCL) studies, participants are presented a number of trials on which causal cues and outcomes are either present or absent. After they have observed some or all of the information, the participants are asked to make a judgment about the causal relation between each cause and the outcome. In one standard design, participants are first exposed to $\mathrm{A}+$ (cue A followed by the outcome) and B- (cue B not followed by the outcome) trials. In a second learning stage, the participants see $\mathrm{AX}+$ (cue A and cue X presented together and followed by the outcome) and BY + trials. Several studies (e.g., Aitken, Larkin, \& Dickinson, 2001; Dickinson \& Burke, 1996) have found that the causal ratings for cue $X$ at the end of the second learning stage are lower than the causal ratings for cue Y. This result is referred to as forward cue competition.

Forward cue competition, however, confounds two different cue competition effects-namely, forward blocking and reduced overshadowing. When a target cue $\mathrm{X}$ is paired together with an alternative cue $\mathrm{A}$ and followed by the outcome $(\mathrm{AX}+)$, causal ratings for cue $\mathrm{X}$ are lower when the $\mathrm{AX}+$ trials are preceded by $\mathrm{A}+$ trials than when no $\mathrm{A}+$ trials are presented. This effect is referred to as forward blocking (see, e.g., Dickinson, Shanks, \& Evenden, 1984; Shanks, 1985). Similarly, when a target cue Y is paired together with an alternative cue $\mathrm{B}$ and followed by

S.V. is a doctoral researcher at the Special Research Fund of Ghent University. We thank Oskar Pineño for a helpful discussion of a previous draft of this article. Correspondence concerning this article should be addressed to S. Vandorpe, Department of Psychology, Ghent University, Henri Dunantlaan 2, B-9000 Ghent, Belgium (e-mail: stefaan.vandorpe @ugent.be). the outcome, causal ratings for cue $\mathrm{Y}$ will be higher when $\mathrm{BY}+$ trials are preceded by $\mathrm{B}-$ trials than when no $\mathrm{B}-$ trials are presented. This effect is referred to as reduced overshadowing (e.g., De Houwer, Beckers, \& Glautier, 2002).

The aim of this study was to compare the relative contributions of forward blocking and reduced overshadowing to forward cue competition. This can be achieved by adding $\mathrm{KL}+$ control trials to the second learning stage of a forward cue competition design (that normally consists of $\mathrm{AX}+$ and $\mathrm{BY}+$ trials only). Just like the target cues $\mathrm{X}$ and $\mathrm{Y}$, the control cues $\mathrm{K}$ and $\mathrm{L}$ are always presented in compound with another cue. The difference between the control and target cues is that the control cues are paired with another cue that has not been presented before, whereas the target cues are paired with a cue previously presented on its own and followed by the outcome (forward blocking) or not followed by the outcome (reduced overshadowing). Within this design, forward blocking can be calculated by subtracting the causal rating for $\mathrm{X}$ from the mean causal rating for K and L. Similarly, reduced overshadowing can be calculated by subtracting the mean causal rating for $\mathrm{K}$ and $\mathrm{L}$ from the causal rating for cue $\mathrm{Y}$.

There are some indications from previous studies that reduced overshadowing may be stronger than forward blocking. Larkin, Aitken, and Dickinson (1998) found that unovershadowing was stronger than backward blocking (but see Wasserman \& Berglan, 1998, for conflicting results). Both unovershadowing (also known as release from overshadowing) and backward blocking refer to cue competition effects similar to reduced overshadowing and forward blocking, respectively, but the order of the presented events is reversed; that is, $\mathrm{AX}+(\mathrm{BY}+)$ trials precede the $\mathrm{A}+(\mathrm{B}-)$ trials in a backward blocking (unovershadowing) design. However, we are unaware of any study that has directly compared forward blocking and reduced overshadowing using discrete outcomes. We do not even know of any study that has included an appropriate control to measure reduced overshadowing. The aim of the present study was to fill these gaps in the literature, because the comparison between forward blocking and 
reduced overshadowing can have important theoretical implications (see Discussion).

\section{METHOD}

\section{Participants}

Eighteen psychology students at Ghent University participated as volunteers. All were native Dutch speakers.

\section{Design, Stimuli, and Materials}

The design is given in Table 1. In the first learning stage, six $\mathrm{A}+$ and six $\mathrm{B}-$ trials were presented. In the second learning stage, $\mathrm{AX}+, \mathrm{BY}+$, and $\mathrm{KL}+$ trials were presented six times each. The sequence of trials was randomized for each participant and learning stage separately. The following six names of foods were used for the different cues (translated from Dutch): mushrooms, kiwi, fish, potatoes, coffee, and tomatoes. The cues were presented as colored pictures of foods (15 cm high, $10 \mathrm{~cm}$ wide) against a white background, with the name of the food under the picture in a black color.

The task was presented on a Pentium I PC with a 15-in. screen, and implemented using a custom made Inquisit program. Six different allocations of the foods to the different cues were used, counterbalanced across participants.

\section{Procedure}

After reading the learning instructions (see the Appendix), the participants pressed a key to start the learning stage. Each learning trial began with the presentation of one or two foods in the center of the screen. After 2,000 msec, either the message "allergic reaction" in a red color or the message "no allergic reaction" in a green color was added at the bottom of the screen. This message stayed on the screen for 3,000 msec, after which both the food and the outcome message were erased. The intertrial interval was 3,000 msec.

After the presentation of all 30 trials, the test instructions appeared on the screen (see the Appendix). During each trial of the test stage, a single food was presented in the center of the screen with a rating scale underneath it. The scale consisted of 10 digits ranging from 1 to 10, with 1 labeled no cause of allergy and 10 labeled cause of allergy. The participants could make their causal rating by clicking with the mouse on a digit of the rating scale. After this click, a white screen was presented for $1,000 \mathrm{msec}$. Then a new scale from 1 to 10 appeared on the screen with 1 labeled very unsure and 10 very sure. On this scale, the participants could indicate how confident they were about their previous causal rating. The confidence ratings were added for exploratory reasons and will not be discussed further. After the participant had entered the confidence rating, a white screen was presented for $1,000 \mathrm{msec}$ before the start of the

Table 1

Design and Causal Ratings for the Different Cues

\begin{tabular}{|c|c|c|c|c|c|}
\hline \multirow[b]{2}{*}{ Contingency } & \multirow[b]{2}{*}{ Stage 1} & \multirow[b]{2}{*}{ Stage 2} & \multirow[b]{2}{*}{ Test } & \multicolumn{2}{|c|}{ Causal Ratings } \\
\hline & & & & $M$ & $S E$ \\
\hline \multirow{2}{*}{$\begin{array}{l}\text { Forward } \\
\text { blocking }\end{array}$} & \multirow[b]{2}{*}{$\mathrm{A}+$} & \multirow{2}{*}{$\mathrm{AX}+$} & A & 9.9 & .08 \\
\hline & & & $X$ & 5.0 & .54 \\
\hline \multirow{3}{*}{$\begin{array}{l}\text { Reduced } \\
\quad \text { overshadowing }\end{array}$} & \multirow{3}{*}{ B- } & \multirow{3}{*}{$\mathrm{BY}+$} & B & 1.2 & .17 \\
\hline & & & Y & 9.4 & .25 \\
\hline & & & K & 5.4 & .27 \\
\hline Control & & $\mathrm{KL}+$ & $\mathrm{L}$ & 5.6 & .24 \\
\hline
\end{tabular}

Note-The letters refer to foods. The "+" stands for occurrence of the outcome and the "-" for nonoccurrence of the outcome. The outcome was an allergic reaction. next test trial. The six different foods were presented in a random order determined separately for each participant.

\section{RESULTS}

Mean causal ratings for the different cues are given in Table 1. As expected, the mean causal rating for cue A was close to 10 and that for cue B close to 1 . We then computed indexes of forward blocking and reduced overshadowing. Forward blocking was computed as the mean of the causal ratings for cues $\mathrm{K}$ and $\mathrm{L}$ minus the causal rating for cue $\mathrm{X}$, and reduced overshadowing as the causal rating for cue $\mathrm{Y}$ minus the mean of the causal ratings for cues $\mathrm{K}$ and $\mathrm{L}$. The measure for reduced overshadowing $(M=3.92, S E=0.42)$ was more than seven times higher than the measure for forward blocking $(M=0.53, S E=$ $0.44)$. A paired samples $t$ test showed that this difference was significant $[t(17)=5.81, p<.001]$. Furthermore, one-sample $t$ tests revealed that forward blocking was not significantly different from zero $[t(17)=1.19, p>.25]$, but that reduced overshadowing was $[t(17)=9.31, p<$ $.001]$.

\section{DISCUSSION}

In this study, we investigated the relative contribution of forward blocking and reduced overshadowing to forward cue competition. The results were very clear. Reduced overshadowing was significantly stronger than forward blocking. This study also provided, as far as we know, the first evidence for reduced overshadowing in HCL when appropriate controls (i.e., KL+) are used.

Many associative models of animal conditioning that could be or have been applied to HCL cannot provide a straightforward account of these results (see, e.g., Denniston, Savastano, \& Miller, 2001; Dickinson \& Burke, 1996; Miller \& Matzel, 1988; Pearce \& Hall, 1980; Rescorla \& Wagner, 1972; Van Hamme \& Wasserman, 1994; Wagner, 1981). Quite opposite to the results presented here, these models all predict robust forward blocking effects. In essence, associative models explain forward blocking either by a selective learning mechanism (see, e.g., Rescorla \& Wagner, 1972) or by a selective expression mechanism in which the associative strengths of the blocked cue X and its comparator cue A are compared (see, e.g., Miller $\&$ Matzel, 1988). Due to the A+ trials in the first learning stage of a blocking design $(\mathrm{A}+, \mathrm{AX}+)$, either cue $\mathrm{X}$ gains little associative strength during the $\mathrm{AX}+$ trials in the second learning stage (selective learning mechanism) or the associative strength of cue $\mathrm{X}$ is much lower than the associative strength of cue $\mathrm{A}$ at the end of the learning stage (selective expression mechanism). As such, associative models predict robust blocking effects. One possible mechanism by which associative models might account for the lack of blocking is mediated learning. Applied to our design, the representation of $\mathrm{X}$ at the test stage could have activated the representation of $\mathrm{A}$ (by the AX+trials in the second learning stage), which in turn activated the representation of the outcome (by the $\mathrm{A}+$ trials in the first 
learning stage). This could explain why ratings for $\mathrm{X}$ were so high. Note that such an explanation implies that there should be a negative correlation between forward blocking and the strength of the within-compound association between $\mathrm{A}$ and $\mathrm{X}$. Existing evidence suggest that this correlation is, however, absent or positive (see, e.g., Melchers, Lachnit, \& Shanks, 2004; Vandorpe, De Houwer, \& Beckers, 2005).

The well-known and often cited Rescorla-Wagner (1972) model and the revision of this model by Van Hamme and Wasserman (1994) also have problems in explaining reduced overshadowing. When $\mathrm{A}+$ and $\mathrm{B}-$ trials precede $\mathrm{BY}+$ trials, cue $\mathrm{B}$ does not gain associative strength during the $\mathrm{B}-$ trials of the first learning stage. As a consequence, cue $\mathrm{B}$, the reduced overshadowing cue $\mathrm{Y}$, and the control cues have equal (zero) associative strength at the beginning of the second learning stage, and reduced overshadowing should not occur. One can argue that cue $\mathrm{B}$ becomes slightly inhibitory in the first learning stage owing to the context, which acquires excitation on the $\mathrm{A}+$ trials. But the context is reinforced on only half of the trials in the first learning stage (i.e., on the $\mathrm{A}+$ but not the $\mathrm{B}-$ trials). Moreover, it is typically also assumed that the salience of the context is lower than the salience of a discrete stimulus. Therefore, excitatory strength acquired by the context and concomitant inhibitory strength acquired by cue $\mathrm{B}$ will be weak at best at the beginning of the second learning stage, and only a small reduced overshadowing effect should occur, if any. Nevertheless, it is difficult to see how this could be stronger than the blocking effect. But even if one assumes that the context gains some considerable associative strength, the associative strength of cue A should be larger. Therefore, it is difficult to see why no strong blocking effects occurred.

Other associative models such as the attentional models of Mackintosh (1975) and Pearce and Hall (1980), Wagner's (1981) SOP model, and the extended comparator hypothesis of Denniston et al. (2001) also do not provide a straightforward account of our results. All these models have built-in mechanisms whereby cue B becomes a poor competing cue for cue $\mathrm{Y}$ on $\mathrm{BY}+$ trials. Hence, they are capable of explaining reduced overshadowing. However, as has already been mentioned, all these models predict robust blocking effects, and it is therefore difficult to see how they can handle the finding of reduced overshadowing that is significantly stronger than forward blocking. Nevertheless, we do not exclude the possibility that the learning parameters of some of these models could be adjusted in such a way that the models would produce stronger reduced overshadowing than forward blocking. Note, however, that such adjustments would be entirely post hoc and might well be incompatible with how the models explain other findings.

Models of causal learning in which a crucial role is assigned to higher order reasoning processes (see, e.g., Cheng, 1997; De Houwer \& Beckers, 2003; Lovibond, 2003; Waldmann, 2000), however, provide a straightforward a priori explanation of the results of this study. In the case of reduced overshadowing $(\mathrm{B}-, \mathrm{BY}+)$, for example, participants can infer on $\mathrm{BY}+$ trials that $\mathrm{Y}$ has to be the cause of the outcome, because they know that B is not a cause of the outcome. Similarly, in the case of forward blocking $(\mathrm{A}+, \mathrm{AX}+)$, participants can infer on the $\mathrm{AX}+$ trials that $\mathrm{X}$ is not a cause of the outcome if they know that $\mathrm{A}$ is a cause of the outcome. They can, however, be sure about the latter inference only if they can verify that $\mathrm{X}$ does not add anything to the effect of $\mathrm{A}$. If the outcome is merely present or absent, this condition is not fulfilled. If $\mathrm{A}$ already causes the outcome on all trials where $\mathrm{A}$ is present, one cannot verify that $\mathrm{X}$ increases the likelihood that the outcome will occur on AX + trials. Participants would thus be unsure about the causal status of cue $X$ while they could be sure about the causal status of cue Y. As a consequence, reduced overshadowing should be significantly stronger than forward blocking.

Additional evidence for this interpretation of our results comes from De Houwer et al. (2002), who also compared reduced overshadowing and forward blocking. This study differed in an important way from all of the other studies about cue competition in HCL. In De Houwer et al.'s third experiment, reduced overshadowing and forward blocking were of the same magnitude. However, the outcome (an explosion of an army tank) was nondiscrete, and it always occurred with a submaximal intensity of 10 out of 20 on $\mathrm{A}+$ and $\mathrm{AX}+$ trials. Because the blocked cue $\mathrm{X}$ did not increase the intensity of the outcome on $\mathrm{AX}+$ trials in comparison with the intensity of the outcome on A+ trials, participants could with certainty infer that cue $X$ was not a cause of the outcome and a blocking effect of comparable magnitude to reduced overshadowing occurred. ${ }^{1}$

Some readers might be surprised that we failed to find a significant forward blocking effect. However, forward blocking in HCL is not as well documented as is often assumed. In most studies on cue competition, forward blocking and reduced overshadowing have been confounded. Although forward blocking has been found in some studies when appropriate controls were used (e.g., Arcediano, Escobar, \& Matute, 2001; Chapman, 1991; Dickinson et al., 1984; Shanks, 1985), several authors have referred to consistent failures to obtain this effect (e.g., De Houwer et al., 2002; Glautier, 2002). Moreover, when blocking has been found the experimental procedure has often contained specific elements such as the requirement that two cues cannot get the same rating (e.g., Chapman, 1991) or that the cue-outcome relation is probabilistic in nature (e.g., Dickinson et al., 1984; Shanks, 1985). Our failure to find forward blocking is thus not atypical. Moreover, we must emphasize that our main finding is not the absence of a forward blocking effect but rather the observation that forward blocking was significantly smaller than reduced overshadowing.

Nevertheless, an anonymous reviewer pointed out that a comparison between forward blocking and reduced overshadowing might make sense only under conditions that allow for significant forward blocking. It is therefore important to note that we replicated the basic finding of this 
study (i.e., reduced overshadowing significantly stronger than forward blocking) in an additional study that involved two conditions of importance for this study (Vandorpe \& De Houwer, in press). In one condition, we used a simple design-namely, $\mathrm{A}+, \mathrm{B}-$ trials in the first learning stage and $\mathrm{AX}+, \mathrm{BY}+, \mathrm{KL}+$, and $\mathrm{VW}-$ trials in the second learning stage. In a second condition, the basic design was the same, but three different foods were assigned to each cue (i.e., three different $\mathrm{A}+$ events, three different $\mathrm{B}-$ events, three different $\mathrm{AX}+$ events, etc.). Importantly, in both conditions we found significant blocking effects; but these effects were significantly smaller than the reduced overshadowing effects. As such, the results of this recent study not only show that the basic finding of this paper is reliable, but also that reduced overshadowing is stronger than forward blocking even when the latter effect is significantly different from zero.

To conclude, this study provides, as far as we know, the first evidence that reduced overshadowing is stronger than forward blocking in HCL when the outcome is merely present or absent (and under these conditions also the first evidence for reduced overshadowing measured against an appropriate control). This result is problematic for certain associative learning models of HCL but is in line with an a priori prediction of higher order reasoning accounts of cue competition in HCL.

\section{REFERENCES}

Aitken, M. R. F., Larkin, M. J. W., \& Dickinson, A. (2001). Reexamination of the role of within-compound associations in the retrospective revaluation of causal judgements. Quarterly Journal of Experimental Psychology, 54B, 27-51.

Arcediano, F., Escobar, M., \& Matute, H. (2001). Reversal from blocking in humans as a result of posttraining extinction of the blocking stimulus. Animal Learning \& Behavior, 29, 354-366.

Chapman, G. B. (1991). Trial order affects cue interaction in contingency judgment. Journal of Experimental Psychology: Learning, Memory, \& Cognition, 17, 837-854.

Cheng, P. W. (1997). From covariation to causation: A causal power theory. Psychological Review, 104, 367-405.

De Houwer, J., \& Beckers, T. (2003). Secondary task difficulty modulates forward blocking in human contingency learning. Quarterly Journal of Experimental Psychology, 56B, 345-357.

De Houwer, J., Beckers, T., \& Glautier, S. (2002). Outcome and cue properties modulate blocking. Quarterly Journal of Experimental Psychology, 54A, 3-25.

Denniston, J. C., Savastano, H. I., \& Miller, R. R. (2001). The extended comparator hypothesis: Learning by contiguity, responding by relative strength. In R. R. Mowrer \& S. B. Klein (Eds.), Handbook of contemporary learning theories (pp. 65-117). Mahwah, NJ: Erlbaum.

Dickinson, A., \& BURKE, J. (1996). Within-compound associations mediate the retrospective revaluation of causality judgements. Quarterly Journal of Experimental Psychology, 49B, 60-80.

Dickinson, A., Shanks, D., \& Evenden, J. (1984). Judgement of act-outcome contingency: The role of selective attribution. Quarterly Journal of Experimental Psychology, 36A, 29-50.
Glautier, S. (2002). Spatial separation of target and competitor cues enhances blocking of human causality judgements. Quarterly Journal of Experimental Psychology, 55B, 121-136.

Larkin, M. J. W., Aitken, M. R. F., \& Dickinson, A. (1998). Retrospective revaluation of causal judgments under positive and negative contingencies. Journal of Experimental Psychology: Learning, Memory, \& Cognition, 24, 1331-1352.

Lovibond, P. F. (2003). Causal beliefs and conditioned responses: Retrospective revaluation induced by experience and by instruction. Journal of Experimental Psychology: Learning, Memory, \& Cognition, 29, 97-106.

Mackintosh, N. J. (1975). A theory of attention: Variations in the associability of stimuli with reinforcement. Psychological Review, 82, 276-298.

Melchers, K. G., Lachnit, H., \& Shanks, D. (2004). Withincompound associations in retrospective revaluation and in direct learning: A challenge for comparator theory. Quarterly Journal of Experimental Psychology, 57B, 25-54.

Miller, R. R., \& Matzel, L. D. (1988). The comparator hypothesis: A response rule for the expression of associations. In G. H. Bower (Ed.), The psychology of learning and motivation: Advances in research and theory (Vol. 22, pp. 51-92). San Diego: Academic Press.

Pearce, J. M., \& Hall, G. (1980). A model for Pavlovian learning: Variations in the effectiveness of conditioned but not of unconditioned stimuli. Psychological Review, 87, 532-552.

Rescorla, R. A., \& Wagner, A. R. (1972). A theory of Pavlovian conditioning: Variations in the effectiveness of reinforcement and nonreinforcement. In A. H. Black \& W. F. Prokasy (Eds.), Classical conditioning II: Current research and theory (pp. 64-99). New York: Appleton.

SHANKs, D. R. (1985). Forward and backward blocking in human contingency judgement. Quarterly Journal of Experimental Psychology, 37B, 1-21.

VAndorpe, S., \& De Houwer, J. (in press). A comparison of cue competition in a simple and a complex design. Acta Psychologica.

VAndorpe, S., De Houwer, J., \& Beckers, T. (2005). The role of memory for compounds in cue competition. Manuscript submitted for publication.

Van Hamme, L. J., \& Wasserman, E. A. (1994). Cue competition in causality judgments: The role of nonpresentation of compound stimulus elements. Learning \& Motivation, 25, 127-151.

WAGNER, A. R. (1981). SOP: A model of automatic memory processing in animal behavior. In N. E. Spear \& R. R. Miller (Eds.), Information processing in animals: Memory mechanisms (pp. 5-47). Hillsdale, NJ: Erlbaum.

WALDMANN, M. R. (2000). Competition among causes but not effects in predictive and diagnostic learning. Journal of Experimental Psychology: Learning, Memory, \& Cognition, 26, 53-76.

Wasserman, E. A., \& Berglan, I. R. (1998). Backward blocking and recovery from overshadowing in human causal judgement: The role of within-compound associations. Quarterly Journal of Experimental Psychology, 51B, 121-138.

\section{NOTE}

1. The Power PC model of Cheng (1997) is an adjustment of the probabilistic contrast model (Cheng \& Novick, 1990) to account for uncertainty about the causal status of the blocked cue $\mathrm{X}$ when the outcome is merely present on $\mathrm{A}+$ and $\mathrm{AX}+$ trials. Therefore, it can explain the results of this study. This model, however, is not adjusted to deal with variations in intensity of the outcome. Therefore, it cannot account for the data of De Houwer et al. (2002). 


\section{Learning Instructions (translated from Dutch)}

This experiment is about how people learn relations between different events. Try to imagine that you are a doctor. One of your patients suffers from allergic reactions after eating certain foods. To discover which foods lead to an allergic reaction, the patient has eaten specific foods on different days and this was followed by a test on whether an allergic reaction occurred. In a moment, you will see the results of these daily allergy tests one by one on the screen. On each trial, you will first see what the patient had eaten that day. On some days, he only ate one food; on other days he ate two different foods. Look carefully each time at what the patient ate that day. You will also receive information about whether the patient showed an allergic reaction or not. Use this information to determine for each food separately whether it leads to an allergic reaction in your patient. Note that if the patient ate two different foods and there was an allergic reaction, you do not know which of the two foods was responsible for the allergic reaction. You nevertheless have to determine for each food separately to what extent it causes an allergic reaction in the patient.

First you will see information about 30 allergy tests. After that, you will have to judge for each food the extent to which you think it is a cause of an allergic reaction in the patient. Notice that only the presented information can help you. The task is to determine to what extent the foods cause an allergic reaction in this specific patient. Your personal experiences with the foods or occasional knowledge about the properties of the foods are not relevant and cannot help you. Only the presented information matters.

\section{Test Instructions (translated from Dutch)}

Now you have to judge for each food separately to what extent it causes an allergic reaction in the patient. You can do this by giving a score between 1 and 10, where a score of "1" means that the food never causes an allergic reaction and a score of " 10 " means that the food always causes an allergic reaction. Thus, the higher your score, the more likely it is that the food causes an allergic reaction in the patient. Each time, you will also be asked how sure you are of your rating. You can express this using a scale from 1 to 10 , where 1 stands for very unsure and 10 for very sure. 